\title{
Non-toxigenic Vibrio cholerae non-01/non-0139 pseudo-bacteraemia in a neonate: A case report
}

\begin{tabular}{|c|c|}
\hline \multicolumn{2}{|c|}{$\begin{array}{l}\text { Authors: } \\
\text { Wentzel B. Dowling }{ }^{1,2} \\
\text { Mené Van der Westhuyzen } \\
\text { Michele Haumann } \\
\text { Kessendri Reddy }{ }^{1,2} \text { (D) }\end{array}$} \\
\hline $\begin{array}{l}\text { Affiliations: } \\
{ }^{1} \text { Division of M } \\
\text { Microbiology } \\
\text { Immunology, } \\
\text { Health Science } \\
\text { Stellenbosch } \\
\text { Cape Town, Sc }\end{array}$ & $\begin{array}{l}\text { dical } \\
\text { and } \\
\text { aculty of } \\
\text { university, } \\
\text { uth Africa }\end{array}$ \\
\hline \multicolumn{2}{|c|}{$\begin{array}{l}{ }^{2} \text { National Health Laboratory } \\
\text { Service, Tygerberg Hospital, } \\
\text { Cape Town, South Africa }\end{array}$} \\
\hline \multicolumn{2}{|c|}{$\begin{array}{l}{ }^{3} \text { Department of Paediatrics, } \\
\text { Paarl Hospital, Cape Town, } \\
\text { South Africa }\end{array}$} \\
\hline \multicolumn{2}{|c|}{$\begin{array}{l}\text { Corresponding author: } \\
\text { Wentzel Dowling, } \\
\text { wbdowling@gmail.com }\end{array}$} \\
\hline \multicolumn{2}{|c|}{$\begin{array}{l}\text { Received: } 07 \text { Nov. } 2020 \\
\text { Accepted: } 21 \text { May } 2021 \\
\text { Published: } 22 \text { July } 2021\end{array}$} \\
\hline \multicolumn{2}{|c|}{$\begin{array}{l}\text { How to cite this article: } \\
\text { Dowling WB, Van der } \\
\text { Westhuyzen M, Haumann M, } \\
\text { Reddy K. Non-toxigenic } \\
\text { Vibrio cholerae non-O1/ } \\
\text { non-O139 pseudo- } \\
\text { bacteraemia in a neonate: A } \\
\text { case report. S Afr J Infect Dis. } \\
\text { 2021;36(1), a263. https://doi. } \\
\text { org/10.4102/sajid.v36i1.263 }\end{array}$} \\
\hline \multicolumn{2}{|c|}{$\begin{array}{l}\text { Copyright: } \\
\text { (C) 2021. The Authors } \\
\text { Licensee: AOSIS. This } \\
\text { is licensed under the } \\
\text { Creative Commons } \\
\text { Attribution License. }\end{array}$} \\
\hline \multicolumn{2}{|l|}{ Read online: } \\
\hline 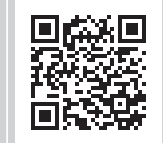 & $\begin{array}{l}\text { Scan this QR } \\
\text { code with your } \\
\text { smart phone or } \\
\text { mobile device } \\
\text { to read online. }\end{array}$ \\
\hline
\end{tabular}

Toxigenic Vibrio cholerae O1/O139 is causative of cholera, which is a well characterised potentially epidemic gastrointestinal disease. Less is known about the pathogenesis and clinical presentation of non-toxigenic $V$. cholerae non-O1/non-O139, although they are increasingly implicated in human disease globally, have been isolated from various South African water sources and can contaminate the environment. The authors describe a case of pseudo-bacteraemia with non-toxigenic $V$. cholerae non-O1/non-O139 in a neonate.

Keywords: Vibrio cholerae; pseudo-bacteraemia; non-toxigenic Vibrio cholerae; non-01/ non-O139 Vibrio cholerae.

\section{Introduction and background}

Toxigenic Vibrio cholerae ( $V$. cholerae) is causative of cholera, a long-established gastrointestinal illness, which remains a potentially epidemic infectious disease with high mortality and morbidity. ${ }^{1,2}$ Vibrio cholerae has a widespread environmental aquatic reservoir and can cause human disease when water sources become contaminated with $V$. cholerae, especially in areas where there is poor sanitation infrastructure, unsafe drinking water, natural disasters or wars. ${ }^{1,2,3}$

The two main pathogenic $V$. cholerae serogroups, $\mathrm{O} 1$ and $\mathrm{O} 139$, have been described extensively in the literature, but less is known about the pathogenesis and clinical presentation of nontoxigenic $V$. cholerae non-O1/non-O139 infections. ${ }^{4,5}$

There are several virulence factors contributing to toxigenic $V$. cholerae pathogenicity: the two major virulence factors are cholera toxin and toxin co-regulated pilus (TCP)., ${ }^{1,2}$ The cholera toxin gene, harboured on a temperate bacteriophage (CTX $\phi)$, can be transmitted horizontally, although the timing of CTX $\$$ lysogeny, integration and replication is complex and poorly understood., ${ }^{1,6,7}$

Compared with the severe diarrhoeal disease caused mainly by the toxin-producing $\mathrm{O} 1$ and $\mathrm{O} 139$ serogroups, non-toxigenic $V$. cholerae non-O1/non-O139 have been reported to cause mild gastroenteritis, wound infections, ear infections, meningitis and bacteraemia. ${ }^{4}$ However, toxigenic $V$. cholerae non-O1/non-O139 such as $V$. cholerae serogroups $\mathrm{O} 75$ and $\mathrm{O} 41$, have also been described as a cause for diarrhoeal disease similar to cholera. ${ }^{8}$ Non-toxigenic $V$. cholerae bacteraemia has predominantly been described in adults with liver pathology (cirrhosis, alcoholism), haematological malignancies, diabetes mellitus and renal disease. ${ }^{5}$

The authors describe a case of pseudo-bacteraemia with non-toxigenic V. cholerae non-O1/ non-O139 in a neonate at a secondary hospital in the Western Cape, South Africa (SA).

\section{Case}

A 1-day old premature neonate was born by normal vertex delivery at 34 weeks' gestation had a birthweight of $2040 \mathrm{~g}$ and Apgar scores of 8 and 9, respectively. Shortly after birth (day 1 of life) the patient developed mild respiratory distress secondary to grade 2 hyaline membrane disease, which was managed with the administration of surfactant and continuous positive air pressure (CPAP) ventilation. Infective markers (done on day 1 of life) included an increased C-reactive protein (CRP) of $53 \mu \mathrm{g} / \mathrm{mL}$ with a normal white cell count of 4.94 cells $\times 10^{9} / \mathrm{L}$. A blood culture and lumbar puncture (LP) were performed on day 1 of life, to exclude sepsis in the context of respiratory distress. The patient was started empirically on ampicillin $150 \mathrm{mg} 6$ hourly and ceftazidime $90 \mathrm{mg} 12$ hourly intravenously on the same day because of a provincial shortage of 
cefotaxime. Following 1 day of CPAP, the patient remained afebrile and was stable on room air. On day 2 of life, the patient developed mild neonatal jaundice and borderline hypoglycaemia, which resolved after treatment with ultraviolet phototherapy and intravenous fluids.

The initial blood culture flagged positive after 48 hour incubation (on day 3 of life) and curved gram-negative bacilli were observed on the Gram stain and processed accordingly (see Microbiology investigation). No bacterial growth was detected, from a repeat blood culture (performed on day 5 of life), following 5 days of incubation. However, the patient was on empiric antibiotics at the time of blood culture collection. The cerebrospinal fluid (CSF) analysis was within normal value ranges. A follow-up CRP, taken on day 6 of life, was within normal limits $(4 \mu \mathrm{g} / \mathrm{mL})$. The patient's antibiotic therapy was de-escalated to ampicillin $150 \mathrm{mg} 6$ hourly and gentamicin $15 \mathrm{mg}$ once daily intravenously on day 3 of life as the patient was clinically stable. A 7-day antibiotic course was completed in total.

The patient received formula milk on day 1 and following successful latching, exclusive breastfeeding was instituted. The patient had no community exposure prior to discharge at day 8 of life. The mother reported no comorbidities, no recent exposure to shellfish, and no history of gastroenteritis. She reported washing the baby with tap water during the hospital stay (from day 1 of life) and consumed unboiled tap water at home. The exact timing of washing of the baby, in relation to the blood culture venepuncture (on day 1 of life), could not be elucidated. Further history of iatrogenic exposure to tap water or washing remains unclear. A stool sample taken from the mother ( 5 days after the baby's birth) excluded the presence of $V$. cholerae.

As a result of the patient's presentation, stable clinical course, absence of features suggestive of neonatal non-toxigenic $V$. cholerae non-O1/non-O139 infection (such as meningoencephalitis) and $V$.cholerae not being endemic in the Western Cape, it was concluded that this was a non-toxigenic $V$. cholerae non-O1/non-O139 pseudo-bacteraemia.

\section{Microbiology investigation}

The blood culture bottle was incubated in the BacT/Alert 3D automated incubator (bioMérieux Inc., Marcy l'Etoile, France) and microbial growth was detected after $48 \mathrm{~h}$ with curved gram-negative bacilli observed on the Gram stain (see Figure 1). The blood culture broth was sub-cultured onto tryptose blood agar, cooked blood agar and MacConkey agar (without crystal violet) and incubated overnight in a $5 \% \mathrm{CO}_{2}$ enriched atmosphere at $35^{\circ} \mathrm{C}$.

Large dry greyish-brown colonies with beta-haemolysis grew on the tryptose blood agar plate (see Figure 2a), with corresponding growth on cooked blood agar and MacConkey agar. Automated biochemical analysis, using the VITEK 2 Gram-negative identification card (bioMérieux Inc., Marcy l'Etoile, France) identified $V$. cholerae with $99 \%$ confidence in identification.
A thiosulfate-citrate-bile salts-sucrose agar (TCBS) and bile aesculin agar were inoculated and incubated overnight (to assist in differentiating $V$. cholerae from Aeromonas species). The TCBS agar showed yellow colonies in keeping with $V$. cholerae (see Figure $2 b$ ), no aesculin hydrolysis was observed and oxidase test was positive. Baltimore Biological Laboratory (BBL) Crystal Enteric/Non-Fermenter identification system (Becton Dickinson Inc., USA) could not differentiate between $V$. cholerae and Aeromonas hydrophila, as these two bacteria are morphologically and biochemically very similar.

Proteomic analysis with the Vitek MS (bioMérieux Inc., Marcy l'Etoile, France) confirmed the identification of $V$. cholerae. The isolate was further tested at the National institute for Communicable Diseases (NICD) Centre for Enteric Diseases, with molecular methods that verified the isolate to be a nontoxigenic $V$. cholerae non-O1/non-O139 serogroup.

\section{Discussion}

Toxigenic $V$. cholerae is endemic in many sub-Saharan African countries. ${ }^{2}$ In SA, sporadic imported cases are reported annually from neighbouring countries such as Zimbabwe. ${ }^{2,9}$ A few major cholera epidemics have been described in SA, with the largest outbreak being in 2008/2009 and only one SA study from the 1980 s describing toxigenic $V$. cholerae bacteraemia in a neonate..$^{2,9,10}$ Toxigenic $V$. cholerae O1/O139 is currently not endemic in the Western Cape with the last known cases coinciding with the 2008/2009 epidemic. ${ }^{9}$ Nontoxigenic $V$. cholerae non-O1/non-O139 is rare in neonates, with only eight previous published cases. ${ }^{11,12,13,14}$ The majority of these cases $(7 / 8)$ presented with meningoencephalitis and had residual neurological deficits following infection. ${ }^{11,12,13,14)}$ In the remaining case, ${ }^{15}$ the patient presented with fever, did not have a LP and demised in hospital.

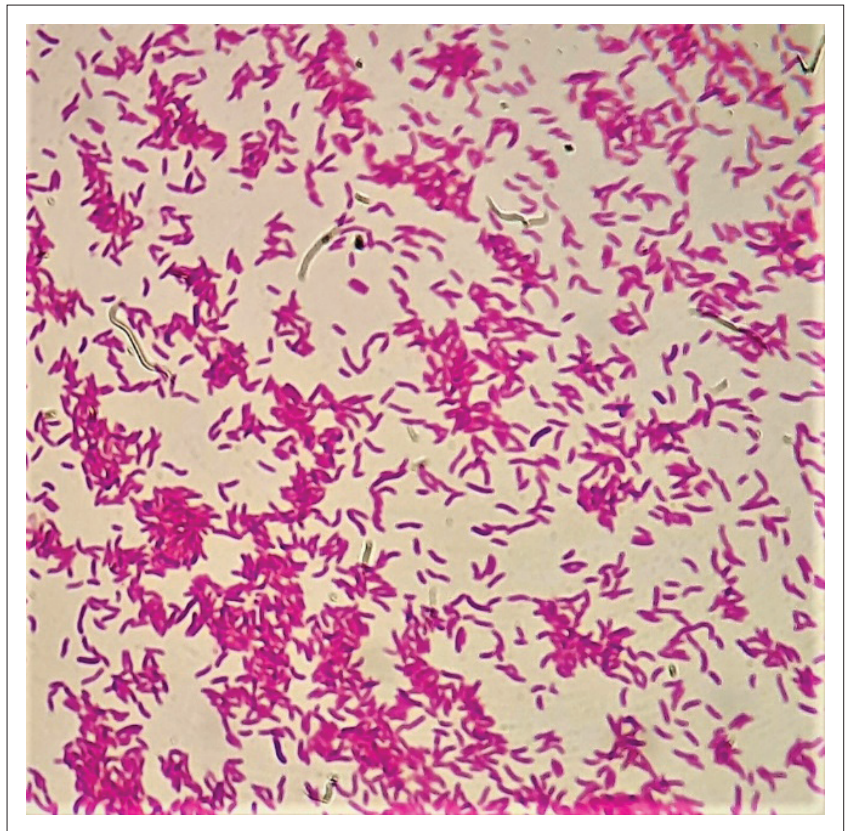

FIGURE 1: Gram stain with comma-shaped Gram-negative bacilli suggestive of Vibrio cholerae. 


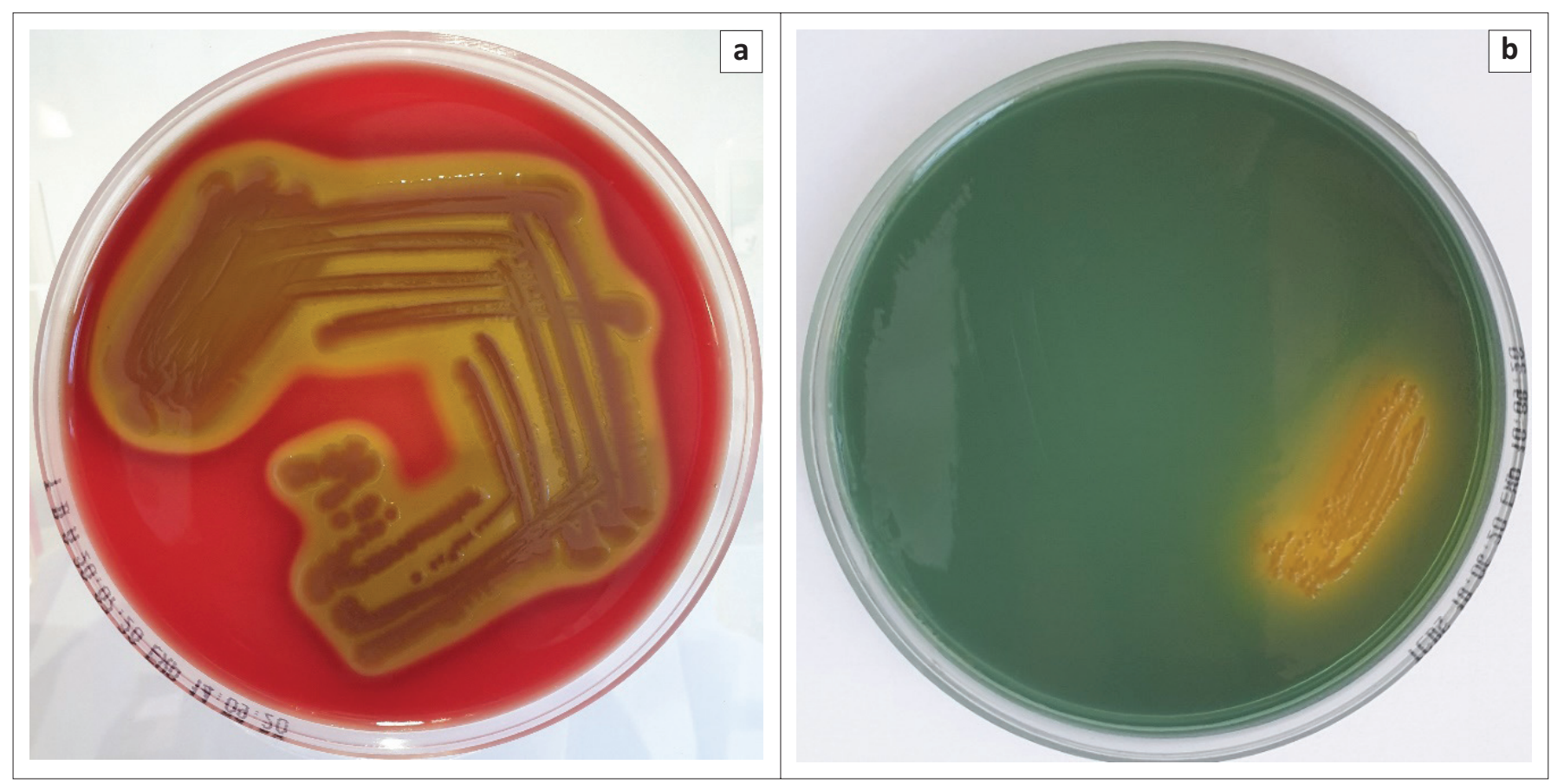

FIGURE 2: (a) Characteristic greyish-brown colonies of Vibrio cholerae with a wide zone of beta haemolysis on tryptose blood agar plate after overnight incubation at $35^{\circ} \mathrm{C}$ in a $5 \% \mathrm{CO}_{2}$ enriched atmosphere; (b) Thiosulphate-citrate-bile salts-sucrose agar with yellow colonies, in keeping with Vibrio cholerae after overnight incubation at $35^{\circ} \mathrm{C}$ in ambient air.

This is in contrast with the clinical findings in our case, where the patient recovered well, had a normal CSF analysis, had a negative repeat blood culture (whilst on empiric antibiotics) and had a repeat CRP that was not elevated.

Our case represents a non-toxigenic $V$. cholerae non-O1/ non-O139 pseudo-bacteraemia and highlights the likely presence of non-toxigenic $V$. cholerae non-O1/non-O139 in water sources from healthcare environments in the Western Cape. It also highlights the complexity of the laboratory diagnosis of non-toxigenic $V$. cholerae non-O1/non-O139, as Vibrio species can be incorrectly identified as the more commonly isolated Aeromonas species because of biochemical similarities. ${ }^{7,16}$ Important laboratory tests to differentiate between $V$. cholerae and Aeromonas species are the observation of comma-shaped Gram-negative bacilli on Gram stain, the presence of yellow colonies on TCBS agar, a positive string test, a lack of aesculin hydrolysis and 'shooting star' motility on wet preparation or darkfield microscopy. ${ }^{16,17}$

It is important to note that only serogroups $\mathrm{O} 1$ and $\mathrm{O} 139$ have been implicated in pandemics ${ }^{7}$ and that these serogroups are distinct from the diverse spectrum of non-toxigenic $V$. cholerae non-O1/non-O139 found in marine and estuarine environments. ${ }^{2}$ Environmental studies in SA have reported both toxigenic (unknown serogroups) and non-toxigenic $V$. cholerae non-O1/non-O139 isolates from various water sources..$^{18,19,20}$ Transformation from non-O1/ non-O139 serogroups to O1/O139 serogroups and toxigenic transformation, has been reported, but is rare in the environment. $^{6}$
In our case study, the source of the $V$. cholerae isolate, causing pseudo-bacteraemia, is poorly elucidated but is likely from an environmental or aquatic reservoir. The most likely source of contamination is thought to be the hospital water supply system. Contaminated water could have been introduced onto the skin either by washing or a breakdown in aseptic technique whilst performing the blood culture.

As both toxigenic and non-toxigenic $V$. cholerae strains have an ubiquitous aquatic niche, the maintenance and monitoring of water and sewerage systems and the prompt notification of clinical cholera disease ${ }^{1,3}$ is crucial to prevent the resurgence of cholera in SA. It is also important to remain vigilant to potential cholera outbreaks, even when non-toxigenic strains are isolated from clinical specimens.

Our case of non-toxigenic $V$. cholerae pseudo-bacteraemia aims to increase awareness of the role of non-toxigenic $V$. cholerae strains in the clinical setting. As cholera is infrequent in SA, it is imperative to ensure continued education on the presentation and laboratory diagnosis of cholera, the diverse clinical presentations (and potential contaminant role) of non-toxigenic $V$. cholerae and the implications of a $V$. cholerae diagnosis on patient treatment and public health response, for both toxigenic and nontoxigenic $V$. cholerae cases.

\section{Acknowledgements}

The authors would like to acknowledge the diagnostic work performed by the staff of Tygerberg Hospital NHLS Medical Microbiology department for this case. 


\section{Competing interests}

The authors declare that they have no financial or personal relationships that may have inappropriately influenced them in writing this article.

\section{Authors' contributions}

W.B.D., M.V.D.W., M.H. and K.R. have all contributed significantly to the work, seen the completed manuscript and approved the content there of for publication.

\section{Ethical considerations}

The authors followed ethical standards for research without contact with human or animal subjects and obtained consent for this case.

\section{Funding information}

This research received no specific grant from any funding agency in the public, commercial or not-for-profit sectors.

\section{Data availability}

Data sharing is not applicable to this article as no new data were created or analysed in this study.

\section{Disclaimer}

The views and opinions expressed in this article are those of the authors and do not necessarily reflect the official policy or position of any affiliated agency of the authors.

\section{References}

1. Ramamurthy T, Mutreja A, Weill F-X, Das B, Ghosh A, Nair GB. Revisiting the global epidemiology of Cholera in conjunction with the genomics of Vibrio cholerae. Front Public Health. 2019;7:203. https://doi.org/10.3389/fpubh.2019.00203

2. Deen J, Mengel MA, Clemens JD. Epidemiology of cholera. Vaccine. 2020;38(1):A31-A40. https://doi.org/10.1016/j.vaccine.2019.07.078

3. Mari L, Bertuzzo E, Righetto L, et al. Modelling cholera epidemics: The role of waterways, human mobility and sanitation. J Roy Soc Interface. 2012;9(67):376-388. https://doi.org/10.1098/rsif.2011.0304

4. Deshayes S, Daurel C, Cattoir V, Parienti J-J, Quilici M-L, De La Blanchardière A. Non-01, non-0139 Vibrio cholerae bacteraemia: Case report and literature review. SpringerPlus. 2015;4(1):575. https://doi.org/10.1186/s40064-015-1346-3
5. Engel MF, Muijsken MA, Mooi-Kokenberg E, Kuijper EJ, Van Westerloo DJ. Vibrio cholerae non-O1 bacteraemia: Description of three cases in the Netherlands and a literature review. Eurosurveillance. 2016;21(15):30197. https://doi. a literature review. Eurosurveillance.
org/10.2807/1560-7917.es.2016.21.15.30197

6. Pang B, Yan M, Cui Z, et al. Genetic diversity of toxigenic and nontoxigenic Vibrio cholerae serogroups $\mathrm{O} 1$ and $\mathrm{O} 139$ revealed by array-based comparative genomic hybridization. J Bacteriol. 2007;189(13):4837-4849. https://doi.org/10.1128/ jb.01959-06

7. Regina LaRocque JBH. Cholera: Microbiology and pathogenesis - UpToDate. UpToDate [homepage on the Internet]. 2018; p. 1-12 [cited 2019 Dec 23]. Available from: https://www-uptodate-com.ez.sun.ac.za/contents/choleramicrobiology and pathogenesis

8. Tobin-D'Angelo M, Smith AR, Bulens SN, et al. Severe diarrhea caused by cholera toxin-producing Vibrio cholerae serogroup $\mathrm{O} 75$ infections acquired in the southeastern United States. Clin Infect Dis [serial online]. 2008 [cited 2020 Aug 18];47(8):1035-1040. Available from: https://academic.oup.com/cid/articlelookup/doi/10.1086/591973

9. Ismail H, Smith AM, Tau NP, Sooka A, Keddy KH. Cholera outbreak in South Africa, 2008-2009: Laboratory analysis of Vibrio cholerae 01 strains. J Infect Dis. 2013;208(Suppl 1):S39-S45. https://doi.org/10.1093/infdis/jit200

10. Coovadia YM, Bhamjee A, Isaacson M, Chb I, Bhamjee FA. Vibrio cholerae bacteraemia newborn infant In a. S Afr Med J [serial online]. 1983 [cited 2019 Dec 23];64(11):405-406. Available from: http://www.ncbi.nlm.nih.gov/pubmed/661 2541

11. Kerketta JA, Paul AC, Balaji V, Kirubakaran C, Jesudason MV, Moses PD. Non-01 Vibrio cholerae septicemia and meningitis in a neonate. Indian J Pediatr. 2002;69(10):909-910. https://doi.org/10.1007/bf02723720

12. Hao $\mathrm{Y}$, Wang $\mathrm{Y}, \mathrm{Bi} \mathrm{Z}$, et al. A case of non-O1/non-O139 Vibrio cholerae septicemia and meningitis in a neonate. Int J Infect Dis. 2015;35:117-119. https://doi. org/10.1016/j.ijid.2015.05.004

13. Baig MZ, Abdullah UH, Shafquat Y, Humayun KN, Zafar A. Non O1, non O139 Vibrio cholerae bacteraemia in an infant; case report and literature review. J Pak Med Assoc. 2018;68(4):650-652.

14. Sudarmana A, Kelly J. Climate change and the spread of disease: An illustrative case of the first Australian invasive non-toxigenic Vibrio cholerae infection in a newborn. J Paediatr Child Health. 2020;57(6):932-934. https://doi.org/10.1111/jpc.15063

15. Sarwar S, Hannan A, Sultana Q, et al. Non-O1Vibrio cholerae bacteremia in an infant, first case report from Pakistan. J Infect Dev Ctries. 2016;10(2):188-189. https://doi.org/10.3855/jidc.6554

16. Public Health England, Standards Unit, Microbiology Services., Identification of Vibrio and Aeromonas species. UK Standards for Microbiology Investigations Bacteriology Identification ID 19; 2015; Issue no: 3. Available from: https://www. gov.uk/ukstandards-for-microbiology-investigations-smi-quality-and-consistencyin-clinical-laboratories

17. Procop GW, Church D, Hall G, et al. Koneman's color atlas and textbook of diagnostic microbiolgy. Philadelphia, PA: Lippincott, Williams \& Wilkins; 2017, Vol. 8, p. 455-457.

18. Okoh Al, Sibanda T, Nongogo V, Adefisoye M, Olayemi OO, Nontongana $N$. Prevalence and characterisation of non-cholerae Vibrio spp. in final effluents of wastewater treatment facilities in two districts of the Eastern Cape Province of South Africa: Implications for public health. Environ Sci Pollut Res. 2015;22(3):2008-2017. https://doi.org/10.1007/s11356-014-3461-z

19. Madoroba E, Momba MNB. Prevalence of vibrio cholerae in rivers of mpumalanga province, south africa as revealed by polyphasic characterization. Afr J Biotechnol [serial online]. 2010 [cited $2021 \mathrm{Ma}$ 28];9(43):7295-7301. Available from: https://www.ajol.info/index.php/ajb/ article/view/130356

20. Okeyo AN, Nontongana N, Fadare TO, Okoh Al. Vibrio species in wastewater final effluents and receiving watershed in South Africa: Implications for public health Int J Environ Res Public Health. 2018;15(6):1266. https://doi.org/10.3390/ ijerph15061266 